\title{
Bayesian Contact Tracing for Communicable Respiratory Disease
}

\author{
Ayman Shalaby* and Daniel Lizotte \\ University of Waterloo, Waterloo, ON, Canada
}

\section{Objective}

The purpose of our work is to develop a system for automatic contact tracing with the goal of identifying individuals who are most likely infected, even if we do not have direct diagnostic information on their health status. Control of the spread of respiratory pathogens (e.g. novel influenza viruses) in the population using vaccination is a challenging problem that requires quick identification of the infectious agent followed by large-scale production and administration of a vaccine. This takes a significant amount of time. A complementary approach to control transmission is contact tracing and quarantining, which are currently applied to sexually transmitted diseases (STDs). For STDs, identifying the contacts that might have led to disease transmission is relatively easy; however, for respiratory pathogens, the contacts that can lead to transmission include a huge number of face-to-face daily social interactions that are impossible to trace manually.

\section{Introduction}

The evolution of novel influenza viruses in humans is a bio- logical phenomenon that can not be stopped. All existing data suggest that vaccination against the morbidity and mortality of the novel influenza viruses is our best line of defence. Unfortunately, vaccination requires that the infectious agent to be quickly identified and a safe vaccine in large quantities is produced and administered. As was witnessed with the 2009 H1N1 influenza pandemic, these steps took a frustratingly long period during which the novel influenza virus continued its unstoppable and rapid global spreading.

In addition to the different vaccination strategies ( e.g. random mass vaccination, age structured vaccination), isolation and quarantining of infected individuals is another effective method used by the public health agencies to control the spreading of infectious diseases. Isolation is effective against any infectious disease, however it can be very hard to detect infectious individuals in the population when:

1. Symptoms are ambiguous or easily misdiagnosed (e.g. 2009 H1N1 influenza outbreak shared many symptoms with many other influenza like illnesses)

2 . When the symptoms emerge after the individual become infec-

\section{Methods}

We developed a dynamic Bayesian network model to process sensor information from users' cellphones together with (possibly incomplete) diagnosis information to track the spread of disease in a population. Our model tracks real-time proximity contacts and can provide public health agencies with the probability of infection for each individual in the model.

For testing our algorithm, we used a real-world mobile sensor dataset with 120 individuals collected over a period of 9 months, and we simulated an outbreak.

\section{Results}

We ran several experiments where different sub-populations were "infected" and "diagnosed." By using the contact information, our model was able to automatically identify individuals in the population who were likely to be infected even though they were not directly "diagnosed" with an illness.

\section{Conclusions}

Automatic contact tracing for respiratory pathogens is a powerful idea, however we have identified several implementation challenges. The first challenge is scalability: we note that a contact tracing system with a hundred thousand individuals requires a Bayesian model with a billion nodes. Bayesian inference on models of this scale is an open problem and an active area of research. The second challenge is privacy protection: although the test data were collected

in an academic setting, deploying any system will require appropriate safeguards for user privacy. Nonetheless, our work llustrates the potential for broader use of contact tracing for modeling and controlling disease transmission.

\section{Keywords}

Outbreak Detection; Syndromic Surveillance; Mobile; Contact Tracing; Bayesian Algorithms

\author{
*Ayman Shalaby \\ E-mail: aymanshalaby11@gmail.com
} tious. 